\title{
The Derivation of Mesenchymal Stem Cells from Human Embryonic Stem Cells
}

\author{
Shelley E. Brown ${ }^{a, b}$ Wilbur Tong ${ }^{a}$ Paul H. Krebsbacha, ${ }^{a}$ \\ ${ }^{a}$ Biologic and Materials Sciences, School of Dentistry, and ${ }^{b}$ Biomedical Engineering, College of Engineering, \\ University of Michigan, Ann Arbor, Mich., USA
}

\section{Key Words}

Human embryonic stem cells • Mesenchymal stem cells .

\begin{abstract}
Human embryonic stem cells ( $\mathrm{hESCs}$ ) hold promise for tissue regeneration therapies by providing a potentially unlimited source of cells capable of undergoing differentiation into specified cell types. Several preclinical studies and a few clinical studies use human bone marrow stromal cells (hBMSCs) to treat skeletal diseases and repair damaged tissue. However, hBMSCs have limited proliferation and differentiation capacity, suggesting that an alternate cell source is desirable, and hESCs may serve this purpose. Here we describe a protocol for the reproducible derivation of mesenchymal stem cells from hESCs (hES-MSCs). The hES-MSCs have a similar immunophenotype to hBMSCs, specifically they are CD73+, STRO-1+ and CD45-, and are karyotypically stable. The derived hES-MSCs are also capable of differentiating into osteoblasts and adipocytes. When the hES-MSCs were genetically modified with the lineage-specific Col2.3-GFP lentivirus and cultured in osteogenic medium, increased GFP expression was detected over time, indicating the hESMSCs have the capacity to differentiate down the osteogenic lineage and had progressed toward a mature osteoblast phenotype.

Copyright $\odot 2008$ S. Karger AG, Basel
\end{abstract} Osteoblasts · Osteoprogenitor cells

\section{Introduction}

The current major goal for human embryonic stem cell (hESC) research is the controlled differentiation into specific progenitor cells for the purpose of replacing or regenerating damaged tissue. Therefore, the ability to obtain large quantities of multipotent cells from hESCs represents a challenge for cell-based therapy and tissue engineering strategies that currently rely on human bone marrow stromal cells (hBMSCs). Within the diverse population of hBMSCs, there exist early progenitor mesenchymal stem cells capable of self-renewal and multilineage differentiation into cell types such as osteoblasts, chondrocytes and adipocytes [Bianco et al., 2003; Wagers and Weissman, 2004]. While hBMSCs make a useful source of osteoprogenitor cells for tissue engineering strategies, they have limited proliferation and differentiation capacity. On the other hand, hESCs, which are able to proliferate indefinitely in vitro, represent a potentially unlimited source of osteoprogenitor cells.

Recent studies have shown that mesenchymal precursors have been derived from hESCs via various isolation

\begin{tabular}{ll}
\hline Abbreviations used in this paper \\
\hline hBMSCs & human bone marrow stromal cells \\
hESCs & human embryonic stem cells \\
hES-MSCs & $\begin{array}{l}\text { human embryonic stem cell-derived mesenchymal } \\
\text { stem cells } \\
\text { OCN }\end{array}$ \\
osteocalcin
\end{tabular}


methods, and the generation of osteoblasts has been achieved in the presence of known osteogenic supplements and coculture with primary bone-derived cells [Sotille et al., 2003; Cao et al., 2005; Ahn et al., 2006; Karp et al., 2006; Olivier et al., 2006; Duplomb, 2007; Lian et al., 2007; Tong et al., 2007]. However, the identification and characterization of a pure osteoprogenitor cell population has yet to be achieved. Osteoprogenitor cells derived from hESCs have tremendous potential, as they can serve as a tool through which one cannot only characterize early bone development and cellular behavior on bone-related biomaterials, but also have application in regenerative medicine.

Therefore, the goal of this study was to derive progenitor cells from hESCs that can undergo differentiation along mesenchymal lineages. These cells are known as hESC-derived mesenchymal stem cells (hES-MSCs). We generated hES-MSCs and relied on functional and morphological criteria to identify them. The criteria included growth on tissue culture plastic, expression of characteristic mesenchymal stem cell surface markers and the ability to undergo multilineage differentiation. Moreover, we were able to transduce the derived hES-MSCs with bonespecific lentivirus Col2.3-GFP in order to track cells undergoing osteoblast differentiation. By isolating hESMSCs, we have the ability to produce a large supply of osteoprogenitor cells that can be genetically modified and used for tissue engineering strategies.

\section{Materials and Methods}

\section{hESC Culture}

The BG01 cell line was obtained from Bresagen Inc. (Atlanta, Ga., USA) and cultured on irradiated mouse embryonic fibroblast feeder layers at a density of $4 \times 10^{5}$ cells/plate $(0.1 \%$ gelatin coated, $60 \mathrm{~mm}$ ). The hESC culture medium consisted of $80 \%$ (v/v) DMEM/ F12, 20\% (v/v) knockout serum replacement, 200 mM L-glutamine, $10 \mathrm{~mm}$ nonessential amino acids (all obtained from Invitrogen, Carlsbad, Calif., USA), 14.3 M $\beta$-mercaptoethanol (Sigma, St. Louis, Mo., USA) and $4 \mathrm{ng} / \mathrm{ml} \mathrm{bFGF}$ (Invitrogen). Cell cultures were incubated at $37^{\circ} \mathrm{C}$ in $5 \% \mathrm{CO}_{2}$ in air and $95 \%$ humidity with medium changes everyday and manually passaged once per week.

\section{Derivation of $h E S-M S C s$}

To induce mesenchymal differentiation, embryoid bodies were formed and cultured in suspension for 7 days with hESC growth medium in low-attachment culture dishes. Then, approximately 70 embryoid bodies/well were plated onto $0.1 \%$ gelatin-coated 6 well plates in the presence of hBMSC growth medium ( $\alpha$-MEM, $10 \%$ FBS, $200 \mathrm{mM}$ L-glutamine and $10 \mathrm{mM}$ nonessential) and $4 \mathrm{ng} /$ $\mathrm{ml}$ bFGF. The outgrowth cells were cultured for up to 2 weeks to reach confluence, then trypsinized and passaged in a ratio of 1:3. Cells were continually passaged into T-75 flasks until a homogeneous fibroblastic morphology appeared. Differentiation into os- teoblasts and adipocytes was performed as previously described, and von Kossa, alizarin red and oil red $\mathrm{O}$ staining was performed according to standard protocols [Barberi et al., 2005].

\section{Col2.3-GFP Lentiviral Infection}

The Col2.3-GFP lentivirus was a kind gift from Dr. Peng Liu (Aastrom Bioscience Inc., Ann Arbor, Mich., USA). Initially, cells were seeded onto 6 -well plates at $10,000 \mathrm{cells} / \mathrm{cm}^{2}$. The following day, cells were transduced with $0.5 \mathrm{ml}$ of Col2.3-GFP lentivirus along with $5 \mu \mathrm{g} / \mathrm{ml}$ polybrene for $4 \mathrm{~h}$ with shaking. Then, complete growth medium was added and cells were further cultured overnight. Approximately 18-24 h later, the medium was aspirated and either control growth medium or osteogenic medium was added. The cells were then cultured for 28 days.

\section{Immunohistochemistry}

Immunofluorescent staining for mesenchymal stem cell surface markers was performed on the hES-MSCs. After cells were fixed for $30 \mathrm{~min}$ with $4 \%$ paraformaldehyde, they were permeabilized for $10 \mathrm{~min}$ with $10 \%(\mathrm{v} / \mathrm{v})$ Triton X-100 in PBS, then washed twice with a serum wash containing $1 \%(\mathrm{v} / \mathrm{v})$ sodium azide, followed by a blocking step containing $0.5 \%(\mathrm{v} / \mathrm{v})$ Triton X-100 and $1 \%(\mathrm{v} / \mathrm{v})$ sodium azide for $30 \mathrm{~min}$ at room temperature. Dilution buffer containing $2 \mu \mathrm{g} / \mathrm{ml}$ polyclonal rabbit anti-human CD45 and CD73, and monoclonal mouse anti-human STRO-1 (Santa Cruz Biotechnology, Santa Cruz, Calif., USA) were added and incubated at $4^{\circ} \mathrm{C}$ overnight. Following incubation, the cells were rinsed twice with serum wash and incubated in the dark with 8 $\mu \mathrm{g} / \mathrm{ml}$ FITC-labeled and Texas red-labeled secondary antibodies for $30 \mathrm{~min}$ and counterstained with DAPI. Cells were then washed with PBS and fluorescence was observed using a Nikon Eclipse TE3000.

\section{Results}

To confirm that our differentiation protocol into mesenchymal progenitor cells from hESCs was efficient, the expression of surface antigens similar to adult mesenchymal stem cells was evaluated. Immunofluorescent staining on hES-MSCs demonstrated that cells were positive for CD73 and STRO-1, and negative for the hematopoietic marker CD45 (fig. 1).

To verify that the derived hES-MSCs can undergo differentiation along mesenchymal lineages, osteogenic and adipogenic differentiation were performed according to standard differentiation protocols previously described [Barberi et al., 2005]. Differentiation of hES-MSCs towards functional osteoblasts was achieved after being cultured in osteogenic medium for 4 weeks (fig. 2a). Tissue mineralization was demonstrated by von Kossa and alizarin red assays. Moreover, RT-PCR analysis showed the expression of bone-specific transcription factor Runx 2 and the extracellular matrix protein osteocalcin (OCN) mRNA, compared to control cells cultured in 

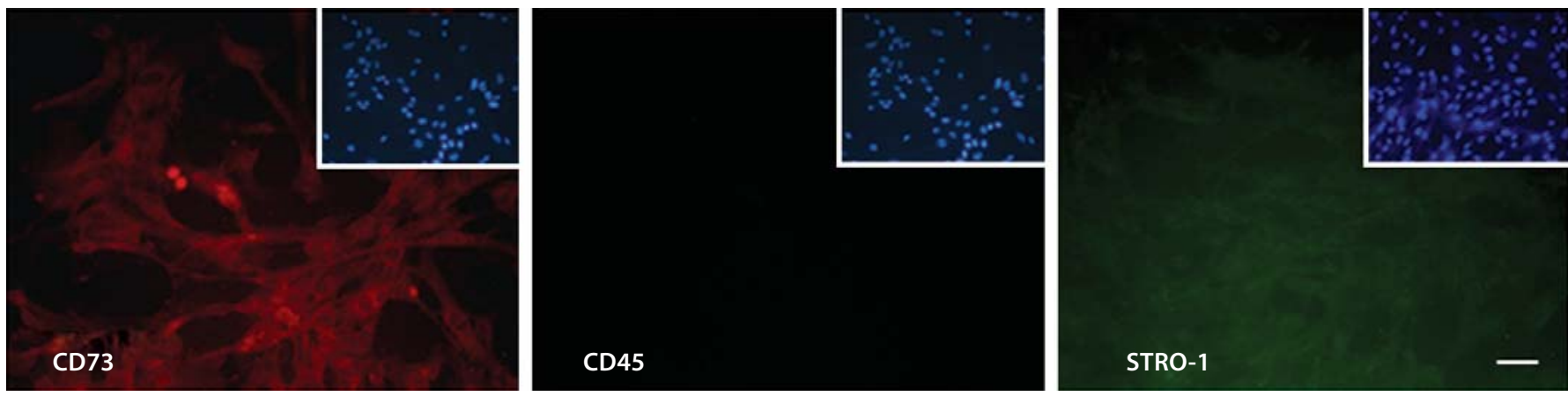

Fig. 1. Characteristic mesenchymal stem cell markers are expressed by derived hES-MSCs. The hES-MSCs are positive for CD73 and STRO-1, but negative for CD45. Insets show DAPI nuclear counterstaining. Scale bar = $100 \mu \mathrm{m}$.
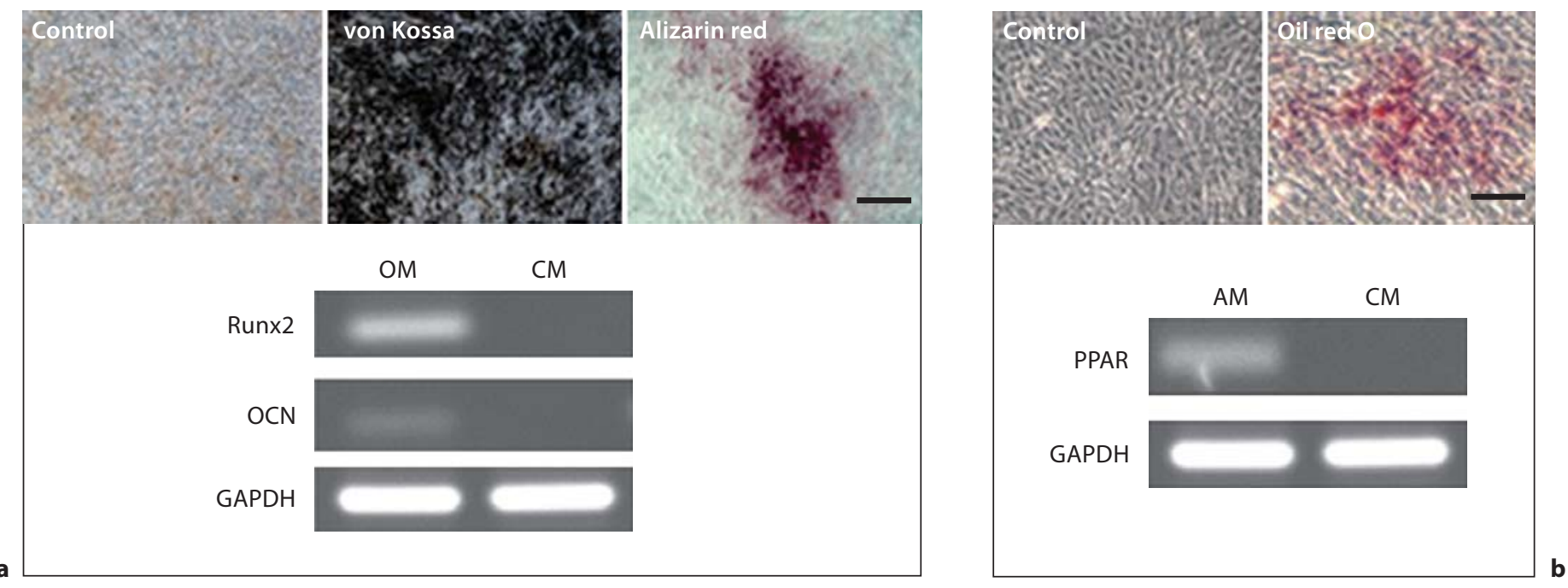

Fig. 2. hES-MSCs undergo differentiation along mesenchymal lineages. a Osteoblast differentiation evidenced by positive von Kossa and alizarin red staining, and Runx 2 and osteocalcin gene expression compared to control. b Adipogenic differentiation shown by positive oil red $\mathrm{O}$ staining and PPAR- $\gamma$ gene expression compared to control. c Karyotype analysis of hES-MSCs showing no chromosomal abnormalities. $\mathrm{OM}=$ Osteogenic medium; $\mathrm{CM}=$ growth medium without osteogenic supplements; $\mathrm{AM}=$ adipogenic medium. Scale bars = $100 \mu \mathrm{m}$.

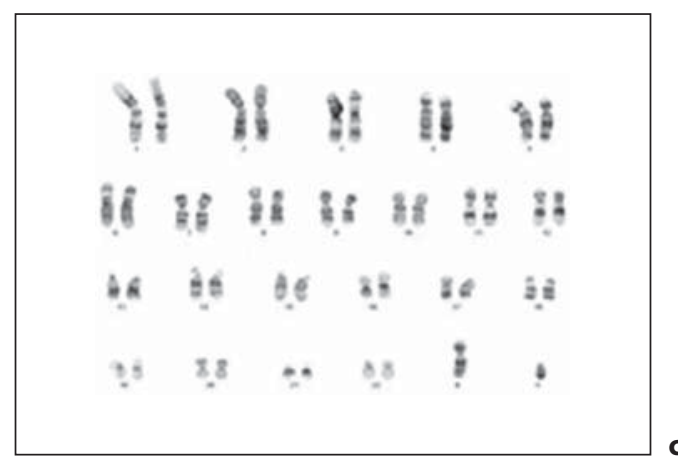

growth medium without osteogenic supplements. Both genes are upregulated in mature osteoblasts.

Differentiation of hES-MSCs towards functional adipocytes was achieved after being cultured in adipogenic medium for 4 weeks (fig. 2b). Positive oil red O staining con- firmed the presence of lipid droplets, and PPAR- $\gamma$ gene expression validated the induction of adipogenesis, compared to control hES-MSCs cultured without supplements.

The ability to genetically modify hESC-MSCs would be quite useful in future tissue regeneration therapies. 

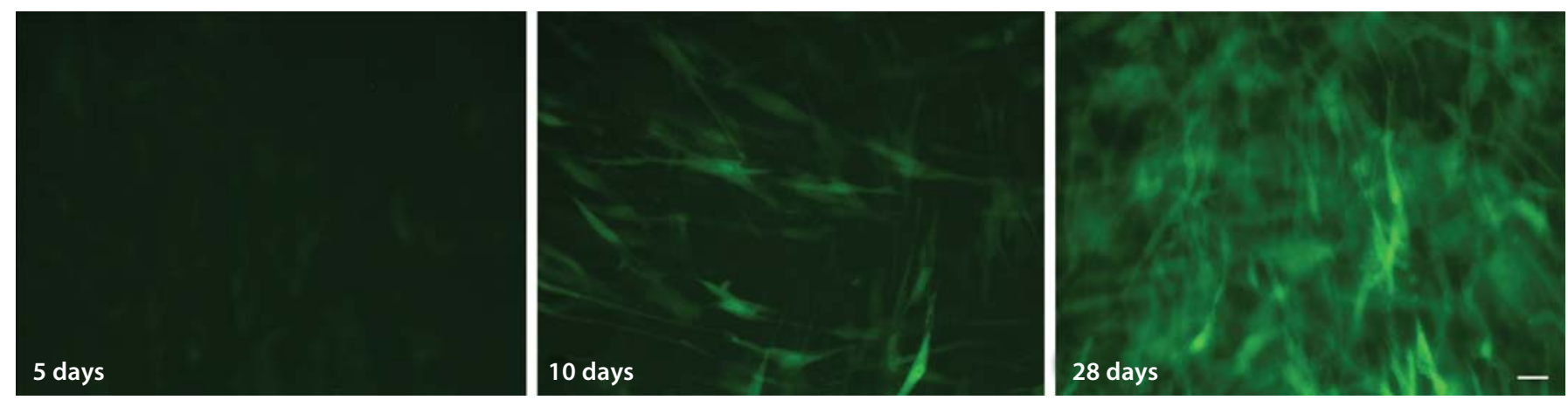

Fig. 3. Commitment to the osteogenic lineage of hES-MSCs transduced with Col2.3-GFP lentivirus. GFP expression increases over time as hES-MSCs differentiate along the osteogenic lineage. hES-MSCs cultured for 5 (a), 10 (b) and 28 days (c) in growth medium with osteogenic supplements. Scale bar $=100 \mu \mathrm{m}$.

hES-MSCs were transduced with the lineage-specific transgene Col2.3-GFP, which is activated as cells differentiate into osteoblasts [Krebsbach et al., 1993]. After viral transfection, hES-MSC were cultured with either normal growth media or osteogenic media over a 28-day time period. In control groups where Col2.3-GFP-transduced hES-MSCs were cultured with normal growth media, no GFP expression was noticed. Meanwhile, a gradual increase in GFP expression was observed during the time course of osteogenic differentiation, as cells progressed towards a mature osteoblast phenotype (fig. 3).

\section{Discussion}

hESC research is a rapidly developing field, and has the potential to impact the medical and scientific community immensely. In general, stem cell research advances the knowledge about how an organism develops and how progenitor cells migrate from the stem cell niche to the site of damaged or diseased tissue. It is vitally important that we begin to explore hESC biology in order to realize the potential of hESCs to cure diseases [Thomson et al., 1998]. Currently, there are major gaps in our knowledge about the growth factors and three-dimensional milieu that influence and direct osteoblast differentiation. The generation of osteoblasts from hESCs has been shown to be successful, as evidenced by osteogenic gene expression of Runx2, bone-specific alkaline phosphatase, and OCN, mineralization confirmed by von Kossa and alizarin red staining, and bone nodule formation [Duplomb, 2007]. One of the first differentiation studies cultured hESCs in the presence of defined osteogenic supplements for 21 days, and was able to demonstrate mineralization and induction of osteoblastic marker expression [Sotille et al., 2003]. hESCs have been cocultured with primary bone-derived cells to induce osteoblast differentiation without the addition of exogenous factors, and cultured in vitro in the presence of known osteogenic factors without the embryoid body formation step - both studies confirming that hESCs have the capacity to differentiate into osteoblasts [Ahn et al., 2006; Karp et al., 2006]. The osteogenic lineages derived from hESCs have tremendous potential and serve as a tool through which we can characterize early bone development and cellular behavior on bone-related biomaterials.

Mineralization assays, RT-PCR analysis and immunofluorescent staining demonstrated that according to our protocol we derived a mesenchymal stem cell population from hESCs. Characterizing the hES-MSCs via immunofluorescent staining demonstrated the expression of cell surface markers that are characteristic of mesenchymal stem cells. The positive expression of CD73 and STRO-1, as well as the lack of expression of CD45 show that the cells possess a similar surface antigen expression profile as hBMSCs. Studies have identified the STRO-1+ fraction of adult hBMSCs as osteogenic precursors, suggesting that we can further isolate osteoprogenitor cells from the derived hES-MSC population [Gronthos et al., 1994, 1999; Stewart et al., 1999].

Functional differentiation results demonstrated the potential of derived hES-MSCs to become osteoblasts and adipocytes. The osteogenic and adipogenic response of hES-MSCs is similar to that of hBMSCs in osteogenic and adipogenic medium [Pittenger et al., 1996]. Von Kossa and alizarin red staining revealed the hES-MSCs formed mineralized bone nodules in culture, and the ex- 
pression of bone-specific transcription factor Runx 2 and extracellular matrix protein OCN suggests the presence of mature osteoblasts. Oil red $\mathrm{O}$ staining revealed the ability of hES-MSCs to differentiate into phenotypically mature adipocyte cells with cytoplasmic lipid droplets. Additionally, the expression of PPAR- $\gamma$ further confirmed differentiation into adipocytes.

In order to track and isolate cells as they undergo differentiation, gene delivery is a useful tool that can be used. The proposed transgene construct is an osteogenic lineage-specific Col2.3-GFP lentivirus, which has been a useful tool for studying hBMSC differentiation [Kalajzic et al., 2002, 2005]. Therefore, we used the Col2.3-GFP transgene to study hES-MSC differentiation along an osteoblast lineage. When the hES-MSCs began as pre-osteoblasts there was low GFP expression, however, there was an increase in GFP expression after 28 days of culture in osteogenic medium, suggesting that hES-MSCs differentiated into mature osteoblasts. The ability to track differentiation allows us to isolate osteoprogenitor cells from the derived hESMSC population.
In summary, although hBMSCs make a useful source of osteoprogenitor cells for tissue engineering strategies, their limited proliferation and differentiation capacity represents an obstacle for therapeutic application. On the other hand, hESCs with their ability to proliferate indefinitely in vitro and multilineage differentiation capacity represent an unlimited source of osteoprogenitor cells. Here we present a protocol for the derivation, identification and isolation of hES-MSCs with osteogenic capacity, which gives us the ability to produce large quantities of genotypically homogenous progenitor cells that can be modified for tissue regeneration strategies.

\section{Acknowledgements}

The authors would like to thank the University of Michigan Human Embryonic Stem Cell Core. This work was funded by the NIH National Institute for Dental and Craniofacial Research (DE016530 and DE016530-01S1). Additionally, the authors would like to thank the University of Michigan Cellular Biotechnology Training Program.

\section{References}

Ahn, S., S. Kim, K. Park, S. Moon, H. Lee, G. Kim, Y. Lee, K. Park, K. Cha, H. Chung (2006) Primary bone-derived cells induce osteogenic differentiation without exogenous factors in human embryonic stem cells. Biochem Biophys Res Commun 340: $403-$ 408.

Barberi, T., L.M. Willis, N.D. Socci, L. Studer (2005) Derivation of multipotent mesenchymal precursors from human embryonic stem cells. PLoS Med 2: e161.

-Bianco, P., M. Riminucci, S. Gronthos, P. Robey (2003) Bone marrow stromal stem cells: nature, biology, and potential applications. Stem Cells 19: 180-192.

Cao, T., B. Heng, C. Ye, H. Liu, W. Toh, P. Robson, P. Li, Y. Hong, L. Stanton (2005) Osteogenic differentiation within intact human embryoid bodies result in a marked increase in osteocalcin secretion after 12 days of in vitro culture, and formation of morphologically distinct nodule-like structures. Tissue Cell 37: 325-334.

Duplomb, L., M. Dagouassat, P. Jourdon, D. Heymann (2007) Concise review: embryonic stem cells: a new tool to study osteoblast and osteoclast differentiation. Stem Cells 25: 544-552.

Gronthos, S., S.E. Graves, S. Ohta, P.J. Simmons (1994) The STRO-1+ fraction of adult human bone marrow contains the osteogenic precursors. Blood 84: 41644173.
Gronthos, S., A.C.W. Zannettino, S.E. Graves, S. Ohta, S.J. Hay, P.J. Simmons (1999) Differential cell surface expression of the STRO-1 and alkaline phosphatase antigens on discrete developmental stages in primary cultures of human bone cells. J Bone Miner Res 14: 47-56.

Kalajzic, I., Z. Kalajzic, S. Clark, A. Lichtler, D. Rowe (2002) Use of Colla1GFP transgenes to identify subpopulations of cells at different stages of the osteoblast lineage. J Bone Miner Res 17: 15-25.

Kalajzic, I., A. Staal, W. Yang, Y. Wu, S. Johnson, J. Feyen, W. Krueger, P. Maye, F. Yu, Y. Zhao, L. Kuo, R. Gupta, L. Achenie, H. Wang, D. Shin, D. Rowe (2005) Expression profile of osteoblastic lineage at defined stages of differentiation. J Biol Chem 280: 2461824626.

Karp, J., L. Ferreira, A. Khademhosseini, A. Kwon, J. Yeh, R. Langer (2006) Cultivation of human embryonic stem cells without the embryoid body step enhances osteogenesis in vitro. Stem Cells 24: 835-843.

Krebsbach, P.H., J.R. Harrison, A.C. Lichtler, C. O. Woody, D.W. Rowe, B.E. Kream (1993) Transgenic expression of COL1A1-chloramphenicol acetyltransferase fusion genes in bone: differential utilization of promoter elements in vivo and in cultured cells. Mol Cell Biol 13: 5168-5174.

Lian, Q., E. Lye, K.S. Yeo, E.K.W. Tan, M. SaltoTellez, T.M. Liu, N. Palanisamy, R.M. El
Oakley, E.H. Lee, B. Lim, S. Lim (2007) Derivation of clinically compliant MSCs from CD105+, CD24-differentiated human ESCs. Stem Cells 25: 425-436.

Olivier, E., A.C. Rybicki, E.E. Bouhassira (2006) Differentiation of human embryonic stem cells into bipotent mesenchymal stem cells. Stem Cells 24: 1914-1922.

Pittenger, M., A. Mackay, S. Beck (1996) Human mesenchymal stem cells can be directed into chondrocytes, adipocytes or osteocytes. Mol Biol Cell 7: 305a.

Sotille, V., A. Thomson, J. McWhir (2003) In vitro osteogenic differentiation of human ES cells. Cloning Stem Cells 5: 149-155.

Stewart, K., S. Walsh, J. Screen, C.M. Jefferiss, J. Chainey, G. Jordan, J.N. Beresford (1999) Further characterization of cells expressing STRO-1 in cultures of adult human bone marrow stromal cells. Jf Bone Miner Res 14: 1345-1356.

Thomson, J., J. Itskovitz-Eldor, S. Shapiro, M. Waknitz, J. Swiergiel, V. Marshall, J. Jones (1998) Embryonic stem cell lines derived from human blastocysts. Science 282: 11451147.

Tong, W., S.E. Brown, P.H. Krebsbach (2007) Human embryonic stem cells undergo osteogenic differentiation in human bone marrow stromal cell microenvironments. I Stem Cells 2: 139-147.

Wagers, A., I. Weissman (2004) Plasticity of adult stem cells. Cells 116: 639-648. 